\title{
Conservation du patrimoine génétique : aspects économiques et institutionnels
}

\author{
PB Joly, M Trommetter \\ Institut national de la recherche agronomique/unité sociologie et économie de la $R \& D$, \\ BP 47, 38040 Grenoble Cedex 09, France
}

Résumé - La confusion des débats préparatoires à la conférence internationale sur la biodiversité laissait présager les difficultés actuelles de sa mise en application. Ces problèmes tiennent pour partie aux carences de l'analyse économique des ressources génétiques : quelle est leur valeur? Comment circulent-elles? Cet article vise à répondre à de telles questions en développant une approche micro-économique de la conservation des ressources génétiques. Ce faisant, il apporte de précieuses indications sur les caractéristiques institutionnelles internationales à recommander pour la régulation de ces ressources.

ressource génétique / incertitude / irréversibilité / valeur d'option / conservation

Summary - The conservation of genetic resources: institutional and economic aspects. The confusion in the preparatory debates at the international conference on biodiversity portended the current difficulties in this field. These problems are partly due to the lack of economic analysis of genetic resources: what is their value? How do they circulate? This article tries to address these questions by developing a microeconomic approach to the conservation of genetic resources. This approach provides useful indications on the characteristics of international institutions to be recommended for the regulation of these resources.

genetic resource / uncertainty / irreversibility / option value / conservation

\section{INTRODUCTION}

L'érosion de la biodiversité a atteint une telle proportion au cours de ces dernières décennies, qu'il y a une prise de conscience générale des risques liés à la destruction massive des forêts tropicales humides et à l'abandon de cultivars locaux et d'espèces sauvages apparentées (érosion de ressources génétiques). La conservation de la diversité génétique peut être réalisée par la conservation in situ, plus communément appelée «préservation de la biodiversité», ou par la conservation ex situ proche de conservatoires de ressources génétiques végétales (RGV). La problématique est 
qu'il existe une érosion de la diversité génétique, qu'il existe des techniques de conservation, mais que le financement accordé est souvent trop faible pour réaliser une conservation efficace. Dans cet article, nous présentons un bref historique des systèmes institutionnels qui ont régi la biodiversité et les ressources génétiques, et leurs justifications économiques et sociales. Ensuite nous définissons une approche de la préservation de la biodiversité et des RGV qui rend mieux compte des stratégies des différents acteurs concernés (conservateurs, utilisateurs...).

\section{LES ÉVOLUTIONS DU CADRE INSTITUTIONNEL}

Les négociations de la convention internationale sur la biodiversité, signée à Rio en juin 1992, furent marquées par la convergence de 2 problèmes d'importance majeure : celui de la conservation et de l'utilisation des ressources génétiques, qui relève pour l'essentiel des problématiques du développement et celui de la préservation de la biodiversité qui correspond à la logique de protection de l'environnement. Un tel rapprochement se fonde sur un élément objectif : les écosystèmes, supports dynamiques de la biodiversité sont également des «réservoirs de gènes» dans lesquels on peut puiser à tout moment. Dès lors, il était évident qu'on ne pourrait parler d'un cadre contraignant pour la préservation de la biodiversité au niveau international sans aborder les questions liées au partage des bénéfices tirés de l'utilisation des ressources génétiques. Cette convergence conduit à associer des problèmes et des entités extrêmement hétérogènes, ce qui explique la difficulté des débats. Cela explique également que la convention soit très difficilement applicable. Le monde de l'utilisation des ressources génétiques et celui de la protection de l'environnement ne se connaissaient pas et fonctionnaient selon des logiques d'actions différentes, fondées sur des systèmes de représentation eux-mêmes très différents. Un seul exemple : alors que dans une logique de conservation des ressources génétiques on cherche à réunir l'ensemble de la diversité génétique de quelques espèces, dans une logique de protection, on essaie de maintenir le plus grand nombre d'espèces différentes. Norton (1987) montre bien par exemple que, alors que les premiers privilégient, finalement, des critères utilitaristes et anthropocentriques, les autres raisonnent sur des valeurs intrinsèques dont un des meilleurs indicateurs réside dans la distance phylogénique. Dans ce dernier cas, on raisonne essentiellement en termes de conservation ex situ (banques de génotypes), dans l'autre en termes de conservation in situ. À ces différences s'ajoutent celles concernant les identités professionnelles (des agronomes dans un cas, des écologues dans l'autre) les institutions et leur histoire (banques de génotypes versus parcs nationaux, FAO versus UNEP...).

Malgré ces difficultés liées à une forte hétérogénéité des cadres de références, tout eût été plus facile si les conditions d'utilisation de la biodiversité ne connaissaient pas elles-mêmes une formidable transformation. Avec le développement des biotechnologies, la biodiversité n'avait plus seulement une valeur pour les populations locales (extractivistes...) ou pour la création d'effets externes locaux ou globaux (l'expression «poumon de la planète» résume bien l'idée). La valeur d'existence ne suffisait plus à donner une appréciation convenable puisque, les conditions d'identification, d'insertion, et d'expression des séquences géniques ayant changé, les écosystèmes peuvent être considérés comme des «réservoirs de gènes». Nous 
reviendrons sur les difficultés d'appréciation de la valeur de ces réservoirs. Ajoutons seulement pour mieux comprendre le problème des institutions internationales que ces utilisations nouvelles imposaient que les problèmes d'appropriation des ressources génétiques fussent posés. On allait alors se trouver confronté à un problème classique : lorsque les techniques d'utilisation ne sont pas réparties de façon homogène, la propriété commune est un vain $\operatorname{mot}^{1}$. Or, non seulement le potentiel de recherche en biologie est concentré dans les pays du nord, mais de plus, si, en toute hypothèse, les négociations actuelles du GATT aboutissaient, ces pays se verraient opposer de nombreux brevets protégeant les inventions biotechnologiques. Dans ce contexte, il était normal qu'une certaine équité soit rétablie en reconnaissant la souveraineté nationale des États sur leurs ressources génétiques. Cette souveraineté était naturellement assortie de l'obligation d'une autorisation préalable à la prospection génétique et d'un partage équitable des bénéfices des ressources génétiques. Ce partage inclut l'accès aux techniques développées à partir des ressources génétiques, ce qui provoqua de vives réactions des entreprises qui travaillent dans le domaine des biotechnologies.

La voie de la privatisation des ressources génétiques (Joly et Trommetter, 1991) n'était bien entendu pas la seule pour résoudre le problème posé. En effet, les débats menés à la FAO depuis la fin des années 1970 avaient progressivement conduit à l'élaboration d'un «système global pour la conservation des RGV». Prenant en compte des éléments essentiels concernant l'utilisation des ressources phytogénétiques, notamment l'importance d'un large accès à la variabilité génétique, ce système s'appuie sur le concept de patrimoine commun de l'humanité $(\mathrm{PCH})$ dont une interprétation particulière s'est progressivement dégagée :

- l'accès aux ressources génétiques est libre mais pas forcément gratuit, ce qui donne la possibilité de protéger la propriété intellectuelle, le Certificat d'obtention végétale $(\mathrm{COV})$ prévoyant le libre accès à la variabilité génétique.

- compte tenu de leur contribution au maintien et à l'enrichissement des ressources génétiques, les agriculteurs détiennent collectivement un droit sur leur utilisation. Ce droit collectif fonde un mécanisme compensatoire, au niveau international. Le Fonds international est supposé jouer ce rôle, avec toutefois les difficultés que nous connaissons.

On a pu craindre que la grande vague de la biodiversité n'emporte ce fragile système élaboré avec patience (Keystone Dialogue, 1991). Les difficultés actuelles de la convention sur la biodiversité et les avancées récentes de la Commission des ressources phytogénétiques de la $\mathrm{FAO}^{2}$ autorisent aujourd'hui un certain optimisme. Il n'en demeure pas moins que les difficultés actuelles révèlent un important déficit de connaissances. Celui-ci provient pour partie de nombreuses carences dans l'analyse économique des ressources génétiques.

1 Les corps célestes sont «Patrimoine commun de l'humanité» (PCH). Pourtant, seules les nations disposant des technologies nécessaires pourront exploiter leurs ressources potentielles.

${ }_{2}$ Avec notamment l'annonce faite par les Centres internationaux de recherche agricole de mettre leurs banques de génotypes sous tutelle de la FAO. 


\section{LES ÉCUEILS TRADITIONNELS DE L'ANALYSE ÉCONOMIQUE DES RGV}

La convention sur la biodiversité s'est intéressée conjointement aux problèmes de préservation de la biodiversité et de conservation des ressources génétiques. Même si les 2 présentent des similitudes au niveau de la valorisation, il est clair qu'il existe des spécificités nécessitant une double approche (tableau I). En effet, la biodiversité possède une valeur économique et sociale mais également environnementale mesurée, traditionnellement, par la volonté de payer des acteurs soit pour pouvoir bénéficier d'un service dans le futur (valeur d'option de Weisbrod en 1964) soit représentant l'attachement à l'existence d'espèces (valeur d'existence) ${ }^{3}$.

Table I. Les différentes valeurs de la biodiversité.

\begin{tabular}{llll}
\hline Mode de conservation & Avantage social & Valeur environnementale & Valeur économique \\
\hline Public & In situ & $\begin{array}{l}\text { Poumon de la planète } \\
\text { Valeur d'existence }\end{array}$ & $\begin{array}{l}\text { Pharmacie } \\
\text { Agronomie, etc }\end{array}$ \\
& Ex situ & & $\begin{array}{l}\text { Amélioration végétale } \\
\text { (cultivars locaux et }\end{array}$ \\
& & $\begin{array}{l}\text { souches sauvages } \\
\text { apparentées) }\end{array}$ \\
& & $\begin{array}{l}\text { Amélioration végétale } \\
\text { et création variétale }\end{array}$ \\
Privé & &
\end{tabular}

Les problèmes liés à la conservation des RGV sont, au départ, issus de l'absence de valeur économique et sociale ${ }^{4}$, puis de l'appropriation de la valeur par les entreprises par le biais des brevets. Le calcul économique sous-jacent pour mesurer l'impact de telles mesures est relativement fruste et a conduit à des surestimations de la valeur des RGV et aux conflits que nous connaissons aujourd'hui.

\section{RGV et technologies}

Par le biais du brevet et du libre accès à la matière première, on tend à donner tout le surplus de la valeur à la technique et rien à la matière première. Or il est clair que la conservation des RGV a des coûts que les décideurs sont prêts à supporter s'il existe un avantage à conserver les ressources génétiques. Comment justifier un financement public de la conservation des RGV si sa valorisation correspond à une logique privée?

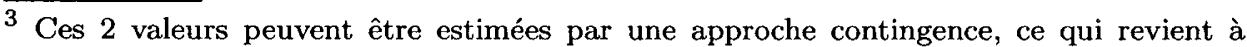
imaginer des situations hypothétiques pour définir la volonté de payer des utilisateurs en fonction des services supplémentaires proposés (Bonnieux et Rainelli, 1992).

${ }^{4}$ Les ressources génétiques ont un statut particulier, car dans la nature, en tant que bien renouvelable, on ne leur accorde aucune valeur économique. 


\section{Effets de substitution}

La valeur des RGV n'est pas uniquement créée par les gènes ou leurs recombinaisons naturelles, elle l'est également par leurs recombinaisons artificielles issues des techniques de l'amélioration végétale. «Un programme du National Cancer Institute a testé 25000 à 35000 espèces de plantes pour détecter leurs activités antitumorales. À peu près 1 espèce sur 1000 s'est avérée avoir des potentialités commerciales. En extrapolant à l'ensemble des plantes du globe, on estime la proportion de plantes ayant des potentialités commerciales en pharmacie entre 1 sur 1000 et 1 sur 10000. D'autre part, des estimations affirment que de 25000 à 75000 espèces disparaîtraient avant l'an 2000. En appliquant le ratio de Principe 1987, ce sont de 3 à 75 espèces à potentialités commerciales qui disparaîtraient. En dollars US actuels, le marché perdu du fait de la disparition de ces 3 à 75 espèces se chiffrerait donc pour l'année 2000 de 2 à 45 milliards, ce qui correspond à une fourchette de PIB qui va de l'Islande à la Finlande.» (Glachant, 1991). L'auteur tire de cette citation que l'indicateur construit est certes une estimation grossière de la valeur d'option, mais qu'elle exprime bien l'ampleur de la catastrophe. Nous ne sommes pas aussi positifs. Cette démarche fait abstraction des possibilités de substitution ${ }^{5}$ entre les ressources naturelles et conduit ainsi à une surestimation de la valeur des RGV. À long terme, les possibilités de substitution entre les espèces sont contingentes à l'état de la technique. Dans une perspective de substituabilité croissante, les processus de standardisation génétique des espèces cultivées vont être accentués. L'évolution des états du monde futurs est lié pour partie aux décisions actuelles, le problème est de nature stratégique : cette situation implique que le décideur construise sa propre vision de l'avenir. En effet, selon le type de plante, la valorisation est différente. Elle dépend du degré d'usage à court/moyen terme, ce qui est très restrictif si l'on veut tenir compte de l'incertitude future. Qu'en sera-t-il quand les transferts interspécifiques seront réalisables?

\section{Appropriation par la ressource}

L'erreur qui consiste à assimiler la valeur de l'innovation à celle de la matière première est relativement courante dans la littérature anglo-saxonne avec Prescott et Allen (1986) et Oldfield (1989) qui, dans la valorisation des RGV d'origine indigène ou sauvage, tiennent peu compte des efforts de $R \& D$ (recherche et développement) qui ont été nécessaires pour parvenir à ce résultat ${ }^{6}$. Ce type

5 "As you would expect, the degree of substituability is also a key factor. If it is very easy to substitute other factors for natural resources, then there is in principle no problem. The world can, in effect get along without natural resources, so exhaustion is just an event, not a catastrophe" (Solow, 1974, p 11). Sans partager entièrement cette citation de Solow, nous retiendrons que la substitution entre les facteurs, même si elle nous écarte de la frontière parétienne, n'est pas forcément une situation si mauvaise.

${ }^{6}$ L'étude de Prescott et Allen est l'une des rares tentatives d'estimation de la contribution des ressources génétiques à la production agricole. L'étude se base sur des travaux d'experts pour repérer les variétés qui utilisent des ressources sauvages et estimer la contribution des «gènes sauvages» à la production (par exemple : un gène de résistance qui permet de réduire les pertes de 30\%). Prescott et Allen évaluent ainsi l'apport des RGA à l'agriculture américaine à 350 millions $\$$ (US\$ de 1980) par an (Oldfield, 1989). 
de démarche a conduit les pays en voie de développement à revendiquer leur souveraineté nationale sur les RGV du fait de sommes qui sont mises en avant, alors que des études tendent à prouver qu'un tel système ne leur serait pas profitable si l'on tient compte de la rémunération des facteurs de production capital et travail (Barton, 1991).

De telles approches renforcent la conservation des espèces en fonction de leur utilité actuelle, ou actuellement anticipée. On favorise ainsi un autorenforcement dans le choix des espèces à préserver. Or les objectifs de la conservation sont avant tout des objectifs de long terme, pour lesquels les anticipations sur les évolutions des techniques et des connaissances sont essentiels. Par la prise en compte, dans l'analyse, de ces incertitudes scientifiques et techniques, on va définir une nouvelle approche (non statique) de la conservation des RGV. Ainsi, il faut séparer la valorisation de la biodiversité et la valorisation des RGV qui n'obéissent pas aux mêmes règles.

\section{UN NOUVEAU CADRE POUR L'ANALYSE DE LA CONSERVA- TION DES RGV}

Au regard des exemples que nous avons donnés dans la section précédente, il semble clair que vouloir donner une valeur économique à la biodiversité et/ou aux RGV est une source de conflits entre utilisateurs et conservateurs et peut même se révéler dangereux pour la justification de la conservation à long terme. Il faut arriver à créer un système incitatif qui soit favorable à la fois à la préservation de la diversité génétique et à la $\mathrm{R} \& \mathrm{D}$. La valorisation des RGV doit, dans ce cas, être formalisée par un modèle séquentiel de décision en avenir incertain.

\section{Les arbitrages économiques}

Notre approche est restrictive par rapport à la conservation de la biodiversité dans son ensemble, pour laquelle une analyse globale et dynamique doit être réalisée (approche $\mathrm{SMS}^{7}$, approche évolutionniste). La préoccupation centrale est la conservation ex situ des RGV face à un environnement économique et social relativement stable $^{8}$. Dans ce cadre d'analyse, l'arbitrage se situe au niveau des ressources conservées. Plus il y a incertitude sur la variation de la qualité demandée (variation de la demande) ou sur l'apparition d'un prédateur et plus il y a un intérêt à rechercher un caractère particulier dans les collections. Cela est à la base de la valorisation des caractères et de l'intérêt de leur conservation. La conservation $e x$ situ du matériel génétique, dans un but économique, est justifiée par :

7 La méthode SMS (Safe Minimum Standard) est apparue à la fin des années 60 . La conservation de la biodiversité est globalement bénéfique à l'humanité. La question essentielle, qu'il s'agisse de la préservation d'une espèce ou d'un habitat naturel, est alors : pouvons-nous assumer les coûts de cette conservation?

8 Par environnement stable, il faut comprendre sans rupture. Les phases de rupture nécessitent une analyse globale et dynamique, alors que nous nous limitons à une analyse locale et dynamique. 
- l'érosion du matériel utilisable (raisons principalement économiques);

- les risques de destruction de grandes étendues de cultures homogènes par la mutation de prédateurs ${ }^{9}$

- l'évolution des techniques de conservation, d'évaluation et d'utilisation (transfert interspécifique) qui permettront de mieux conserver, d'évaluer et surtout de transférer plus rapidement (et de façon fiable) un gène de résistance (ou autre) dans les variétés modernes. L'impact des biotechnologies, en amont, doit permettre d'augmenter les échanges interspécifiques. De ce fait, les espèces ne sont plus conservées pour elles-mêmes, mais pour les caractéristiques qu'elles contiennent (réservoir de gènes).

Quelles sont les différentes options disponibles pour le décideur?

- conserver ou non les RGV : cet arbitrage dépend de l'incertitude sur l'utilisation future des ressources génétiques et sur la mutation des prédateurs ou l'évolution de la demande (de la qualité);

- conserver mais comment?

- diversifié ou évalué (conservation en banque contre core collection) : si un prédateur apparaît et si les techniques d'évaluation n'évoluent pas favorablement, la core collection (décision irréversible en terme de perte de diversité) peut être préférée;

- statique (banque, core collection) ou dynamique : la conservation dynamique permet de créer des nouvelles options dans le futur (nouvelles caractéristiques), que l'on ne pourra découvrir (information) que si la décision de conservation dynamique est prise.

\section{Le cadre théorique}

Avec Weisbrod (1964), les modèles environnementaux prennent une autre dimension. Ils tiennent compte de l'incertitude relative aux états du monde futur et de l'irréversibilité de la décision de développement d'un écosystème. Une telle approche a été approfondie par Henry (1974) et Arrow et Fisher (1974). Cette formalisation dynamique permet de mieux rendre compte de l'idée de Weisbrod, en définissant la valeur d'option (quasi-option value) comme le montant auquel un décideur est prêt à renoncer en première période pour ne pas prendre la décision irréversible et bénéficier ainsi de l'information supplémentaire qui va arriver au cours du temps. Cette valeur d'option est positive, y compris dans le cas d'un décideur neutre au risque.

La limite que nous avons développée sur ces modèles concerne l'irréversibilité de la décision. Ainsi, une irréversibilité apparaît lorsque la décision considérée rend les conditions initiales de la période suivante plus contraignantes. On peut alors définir 2 types de flexibilités compatibles entre elles, une flexibilité diachronique (intertemporelle) et/ou une flexibilité synchronique (intratemporelle). La première reste proche de la définition retenue par Henry. En revanche, la seconde permet de prendre en compte le fait que la réalisation d'un état de la nature peut limiter les actes futurs possibles. Ainsi, une décision peut être moins flexible en terme

\footnotetext{
${ }^{9}$ Les gènes ou pool de gènes résistants se trouvent en général dans les espèces primitives ou sauvages.
} 
temporel, mais créer de nouvelles options en seconde période non disponibles immédiatement pour la décision réversible, ou simplement être plus adaptable à la réalisation de certains événements - la décision irréversible possède une flexibilité à la Stigler (1939) ou plus simplement un gain par l'apprentissage (learning by doing or using $)^{10}$.

\section{UN NOUVEAU CADRE POUR L'ANALYSE DE LA CONSERVA- TION DES RGV}

Le modèle développé par Trommetter (1993) part de la constatation que les coûts de la conservation sont certains, mais que la valorisation est aléatoire et décide le niveau de financement. C'est pourquoi dans un premier temps et grâce à une enquête auprès d'experts, nous avons pu comparer les coûts minimaux pour réaliser une conservation efficace aux financements actuellement accordés aux centres de conservation, après quoi nous avons construit un modèle coûts/avantages/risques en dynamique qui permet de définir une typologie des différents types de conservation en fonction des scénarios d'évolution.

\section{L'analyse des coûts de la conservation}

Grâce à une enquête auprès d'experts, nous avons pu réaliser une étude détaillée des coûts de la conservation des RGV (tableau II). Les coûts de la conservation tels qu'ils ont été construits sont supérieurs aux financements actuels. Ce sont vraisemblablement les coûts minimums nécessaires pour réaliser une conservation viable dans le cas de la banque, et efficace dans le cas de la core collection.

On peut noter que les financements accordés sont fonction de l'anticipation des décideurs sur l'utilisation potentielle des ressources dans les programmes de sélection végétale et sont également fonction du système institutionnel en place.

Tableau II. Financement actuel et coût minimum de la conservation.

\begin{tabular}{|c|c|c|c|c|}
\hline Mode de conservation & $\begin{array}{l}\text { Banque en } \\
\text { champs }\end{array}$ & $\begin{array}{l}\text { Banque de } \\
\text { graines }\end{array}$ & $\begin{array}{c}\text { Core } \\
\text { collection }\end{array}$ & $\begin{array}{c}\text { Gestion } \\
\text { dynamique }\end{array}$ \\
\hline $\begin{array}{l}\text { Nombre d'échantillons } \\
\text { Coûts }\end{array}$ & 8000 & 10000 & 2500 & 1500 \\
\hline \multicolumn{5}{|l|}{ Fonds actuel (1000 FRF) } \\
\hline Fixe & 4000 & 3100 & & \multirow[b]{2}{*}{3000} \\
\hline Variable & 2500 & 800 & & \\
\hline \multicolumn{5}{|l|}{ Coût minimum } \\
\hline Fixe & 4000 & 3100 & 550 & \multirow[b]{2}{*}{3000} \\
\hline Variable & 2500 & $\frac{\text { auto }}{\text { allo }}$ & $\frac{\text { auto } \mid \text { allo }}{950 \mid 1100}$ & \\
\hline
\end{tabular}

Source : Trommetter (1993).

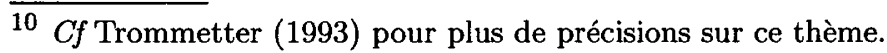




\section{La modélisation utilisée}

L'analyse de ces problèmes nécessite l'utilisation de modèles séquentiels de décision en incertitude, en particulier les modèles à valeur d'option et leurs prolongements. Il s'agit de définir des arbitrages (quelles espèces conserver et comment?) en présence : d'incertitude (apparition d'opportunités favorables, ou défavorables), d'irréversibilité avec la perte d'une caractéristique par la disparition de la plante dans laquelle elle se trouve, et d'information croissante sur les gains ou les pertes en bénéfices nets.

Cette analyse coût/avantage/risque intègre l'incertitude sur : l'apparition d'un nouveau prédateur ou la modification du marché (variation de la demande, de la qualité); l'apparition d'une nouvelle technique de conservation, d'identification, d'évaluation ou de transfert. De plus, dans ce modèle, on tient compte des possibilités de substitution entre les caractères (si un gène important n'existe pas dans une collection, le revenu espéré futur, du fait de la substituabilité, décroit mais bute sur un seuil minimal).

Dans l'étude de la conservation, cela conduit à construire des arbres de décision qui incorporent les anticipations d'apparition d'un prédateur mutant et les évolutions technologiques tant sur les techniques de conservation que sur les techniques d'utilisation. Le financement de ces conservatoires et l'utilisation du matériel conservé sont alors contingents à l'information diffusée par la banque auprès des sélectionneurs. Deux types de risques sont envisagés : le risque temporel de perdre de la diversité génétique (disparition irréversible d'une variété) et le risque de délais de réaction trop importants dans l'utilisation des RGV. L'irréversibilité temporelle est donc un facteur important de la décision, mais elle peut dans bien des cas être plus que compensée par l'existence d'une flexibilité endogène pour la décision plus irréversible temporellement.

\section{Principaux résultats}

L'arbitrage entre conservation et non-conservation, ainsi que le type de conservation qu'il convient de choisir, est fonction de la date moyenne d'apparition d'un prédateur, des probabilités de présence d'un gène de résistance (flexibilité externe), de la date moyenne d'apparition d'une nouvelle technique d'évaluation et de transfert et du degré de substituabilité entre les caractères.

Malgré des financements supérieurs à ceux d'aujourd'hui, en fonction de la date moyenne d'apparition du prédateur, la valeur sociale nette (conservation moins non-conservation) est positive et représente une volonté sociale de financer encore davantage les collections, par conservation d'échantillons supplémentaires (accroissement de la probabilité de trouver un gène de résistance en collection). Dans ce cas, l'effet irréversibilité de Henry est vérifié et devrait entraîner un effort de conservation encore plus important (nombre d'échantillons conservés).

Le choix entre les différentes techniques montre que l'intérêt de la flexibilité externe (probabilité de trouver un gène de résistance plus fort) est réduit par le degré de flexibilité interne inhérent à la technique utilisée (délai d'adaptabilité à la modification de l'environnement). Cet arbitrage donne des résultats très différents selon le cadre d'analyse. La décision moyennement irréversible (core collection) qui 


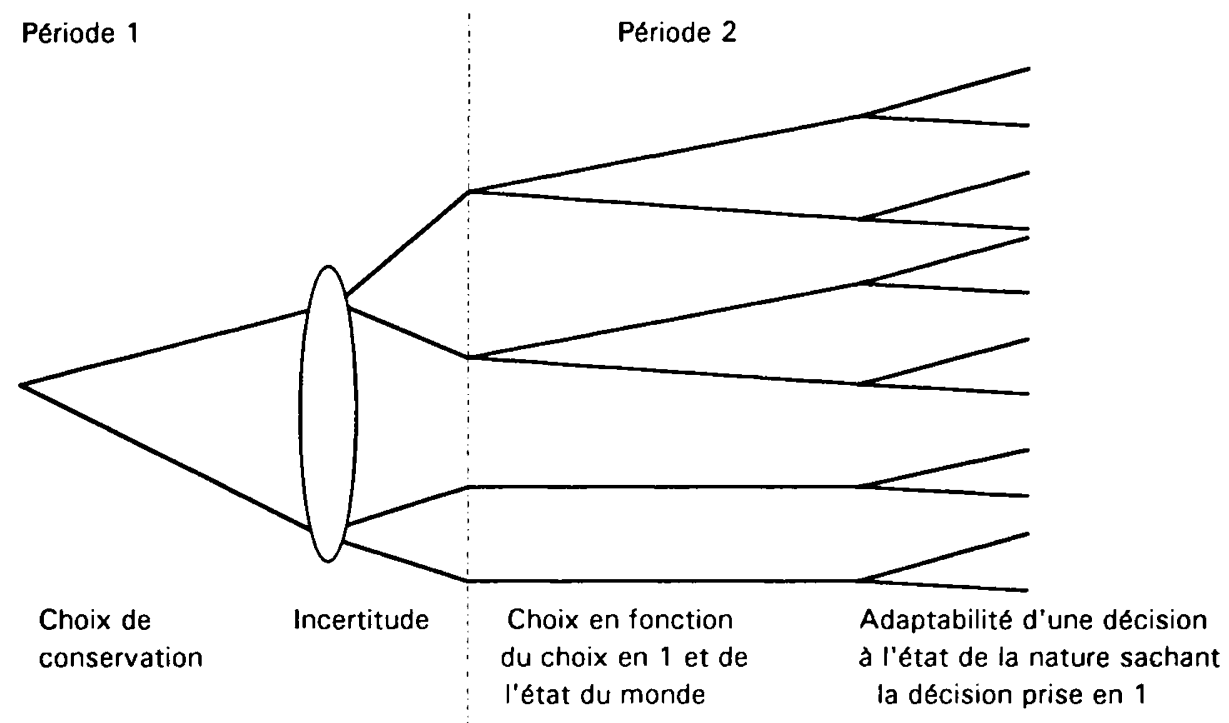

Fig 1. L'arbre du processus de décision.

ouvre de nouvelles options dans le futur peut être préférable à la décision flexible la banque - (même si cette dernière est préférable tant que le prédateur n'apparaît pas). Le fait de considérer que cette décision ouvre des options (adaptabilité plus forte à la réalisation d'un état de la nature) plus intéressantes dans le futur par rapport à la décision flexible, peut entrainer un retournement de la solution classique de l'effet irréversibilité.

Ces arbitrages peuvent être étudiés indépendamment, mais ils sont avant tout interdépendants. L'intégration de la gestion dynamique modifie-t-elle la typologie des techniques de conservation précédemment mise en évidence et les surcoûts apportés par l'association d'une gestion dynamique sont-ils justifiés? Autrement dit, le gain issu de la plus grande probabilité de trouver un gène résistant estil supérieur aux coûts qu'elle entraîne (la valeur étant fonction de la probabilité d'apparition d'un prédateur, de la probabilité de trouver un gène de résistance et de la probabilité de modification des techniques d'évaluation - biologie moléculaire -).

\section{QUEL SYSTĖME INSTITUTIONNEL RECOMMANDER?}

Le décideur face à de tels résultats peut ainsi décider le niveau de financement qu'il est prêt à accorder à la conservation des RGV tout en sachant en contrepartie le niveau de risque que cela fait courir à la société et en particulier la perte en valeur sociale pour la société. Ainsi, nous n'essayons pas de donner une valeur économique aux RGV, mais nous montrons leur utilité potentielle pour la société dans son ensemble. En terme institutionnel cela revient à favoriser le statut de PCH pour les RGV et le brevet avec licences de dépendances ou COV amélioré pour la $R \& D$, le tout se faisant sous les auspices d'un organisme international gérant un 
fonds international pour inciter les pays à conserver les RGV et à préserver la biodiversité. La contribution à ce fonds, si elle est volontaire, sera fonction de la volonté de payer des décideurs nationaux. Le décideur doit ainsi prouver que les coûts de la préservation sont insupportables pour renoncer à le faire ${ }^{11}$.

Cela revient à intégrer une valorisation marchande à des mécanismes compensatoires. Dans un tel cas, les États du Sud auront une incitation plus grande à la conservation de la biodiversité et les États du Nord une incitation plus grande au financement d'actions de préservation de zones en danger ${ }^{12}$.

\section{CONCLUSION}

L'analyse économique de la préservation de la biodiversité et de la conservation des ressources génétiques, telle qu'elle était pratiquée, restait très réductrice de la réalité des ressources génétiques. Pour certains, la justification de la conservation des RGV ne pouvait passer que par sa valorisation économique. Malheureusement des analyses trop frustes, sans prise en compte des possibilités d'évolution rapide des connaissances, ont conduit aux résultats inverses, si l'on en croit les débats contradictoires sur la brevetabilité du vivant, sur la souveraineté nationale des pays sur les ressources et sur le statut des conservatoires ex situ de RGV.

L'approche développée dans cet article est toute autre. Elle tient compte des risques de ne pas conserver les ressources génétiques. La justification économique de la conservation des RGV est donnée par la mesure de la non-perte en valeur sociale suite à l'apparition aléatoire d'une catastrophe naturelle et/ou artificielle. Sur la base d'un modèle séquentiel de décision nous avons ainsi défini (grâce aux études sur les coûts et les évolutions techniques) des hiérarchies sur les différents systèmes de conservation, celles-ci étant contingentes aux scénarios d'évolution construits (apparition de prédateurs, évolucions technologiques, substituabilité des ressources). Le niveau de financement accordé à la conservation des RGV est fonction des risques que le décideur est prêt à accepter au nom des générations présentes et futures.

\section{RÉFÉRENCES}

Arrow KJ, Fischer AC (1974) Environmental preservation, uncertainty, and irreversibility. QJ Econ 88, 312- 319

Arthur W (1988) Competing technologies: an overview. In: Dosietalii. Technical change and economic theory. Pinter Publishers, New York

Barton JH (1991) Relating the scientific and the commercial worlds in genetic resources negociations. International symposium and experts workshop on property rights, biotechnology and genetic resources IFIAS Nairobi, 10-15 juin 1991

11 Pour détruire une espèce, il revient au décideur de montrer que les avantages de cette destruction sont supérieurs aux inconvénients.

12 En effet, la conservation de la biodiversité relevant à la fois de normes et de prix, les pouvoirs publics seront moins réticents vis-à-vis d'un financement, dont les charges sont réparties entre le public et le privé. 
Bonnieux Rainelli P (1991) Catastrophes écologiques et dommages économiques : problèmes d'évaluation à partir de l'Amoco Cadiz. INRA, Editions-Economica Dixon RA, Paiva NL (1991) Prospects for accessing DNA banks for the isolation of genes encoding biological active proteins. In: Conservation of plant genes: DNA banking and in vitro biotechnology. (Adams RP, Adams JE, eds). Academic press, San Diego, 99-118

Glachant M (1991) La biodiversité biologique végétale : éléments d'économie. CERNA, Paris

Henry C (1974) Investment decision under uncertainty: the irreversibility effect. Am Econ Rev 64, 1006-1012

Joly PB, Trommetter M (1991) World regulation and genetic resources: is the model of common heritage sustainable? International symposium and experts workshop on property rights, biotechnology and genetic resources, IFIAS Nairobi, 10-15 juin 1991 Keystone Dialogue (1991) Final consensus report, Oslo Plenary Session. Oslo, Norvège

Llerena P (1985) Décision avec incertitude et irréversibilité : fondements de la théorie de la valeur d'option et application aux investissements productifs. Thèse d'état, Université Louis-Pasteur, Strasbourg

Norton (1987) Why preserve natural variety? Princeton University Press, Princeton Oldfield ML (1989) The value of conserving genetic resources. Sunderland, Sinauer Prescott-Allen R (1986) The first resource: wild species in the North American Economy. Yale University Press New Haven, CT,

Solow RM (1974) The economic of resources or the resources of economics. Am Econ Rev 64, 1-14

Stigler G (1939) Production and distribution in the short run. J Polit Econ 47, 305-327

Trommetter M (1993) Rationalisation économique de la conservation des ressources génétiques végétales. Thèse de doctorat d'Université, Grenoble

Weisbrod BA (1964) Collective consumption services of individual consumption goods. QJ Econ 78, 471-477 